\title{
Beschichtete Werkzeuge mit Diamanten für die CFK-Bearbeitung
}

CFK ist nicht nur ein höchst leistungsfähiges, sondern vor allem auch ein schwer zu zerspanendes Material. Eine wirtschaftliche Bearbeitung ist oftmals nur mit beschichteten Vollhartmetallwerkzeugen möglich. Aktuelle Diamant-Multilayer der CCDia-Reihe gewährleisten laut CemeCon die präzise Bearbeitung von CFK und ein langes Werkzeugleben.

Bei der spanenden Bearbeitung verursachen die harten Kohlenstofffasern einen extrem hohen Abrasionsverschleiß, da helfen nur Schneidstoffe und Beschich- tungen höchster Härte. Vor allem auch, um die Qualitäsanforderungen der Luftfahrtunternehmen zu erfüllen.

CemeCon-Diamant-Multilayer-Beschichtungen haben sich nach Angaben des Unternehmens bereits vielfach bewährt: Beim Fräsen von CFK seien gegenüber einer AlTiN- oder AlCrN-Beschichtung bis zu achtfache Standzeiten möglich. „Die Ergebnisse beim Fräsen eines mehrlagigen CFK-AI-Verbundwerkstoffs zeigten gegenüber einem unbeschichteten Werkzeug sogar noch beeindruckendere
Werte. In diesem Fall konnte die Standzeit dank unserer CCDiaFiberSpeed-Diamantbeschichtung über $900 \%$ verlängert werden“, heißt es. Auch die von der Flugzeugbranche geforderten hohen Präzisionen in der Bohrungstoleranzen $\mathrm{H} 7$ oder $\mathrm{H} 8$ seien mit speziell angepassten Diamantschichten kein Problem.

Die Vorteile der Beschichtung mit Diamant-Multilayern sieht der Hersteller in einer exzellenten Haftung, sehr glatten Oberflächen, extremer Härte und optimaler Schichtspannungsverhältnisse.

\section{ZWICK MEHRKANALIGES MESSSYSTEM FÜR DIE FEDERPRÜFUNG}

Federn sind eines der wichtigsten klassischen Konstruktionselemente und dienen der Energiespeicherung und Energieumformung. Hierbei werden die elastischen Eigenschaften der Werkstoffe genutzt. Ohne Federn, ganz gleich in welcher Bauform, würde kein mechanisches oder mechatronisches System funktionieren. Die unterschiedlichen Einsatzgebiete der Federn erfordern eine besondere Form der Qualitätskontrolle. Die Firma Zwick bietet mit ihrer Mehrkanal-Federprüfvorrichtung eine spezielle Lösung zur Materialprüfung an.
Mit mehrkanaligen Messplattformen kann der Kraftwirkungslinienverlauf von Druck- Schraubenfedern nach Angaben des Ulmer Unternehmens ermittelt werden. Um das zu erreichen, werden Standard-Prüfsysteme mit einer Sechs- oder Neun-Komponenten-Kraftmessplattform ausgerüstet. Diese ermöglichen eine spezifische Ermittlung der Feder-Durchstoßpunkte und der resultierenden Kraft aus den Kraftkomponenten, die bei Belastung der Feder unter Druckkraft entstehen - Kennwerte, die unter anderem wichtig sind, um die Quali- tätsattribute Reibung, Abnutzung und Lebensdauer zu beurteilen.

DieMehrkanal-Federprüfvorrichtung ist in eine Allround-Line-Material-Prüfmaschine eingebaut. Die zwei Prüfräume ermöglichten die Durchführung von Zug- und Druckversuchen in einer Maschine. Der Wegfall des Umrüstaufwands garantiert laut Zwickzum einen nachvollziehbare Prüfergebnisse, da immer mit dem gleichen Aufbau geprüft werde. Zum anderen ermöglichten die beiden Prüfräume eine erhebliche Zeitersparnis. Die Prüfmaschine ist bis Fmax 250 kN verfügbar.

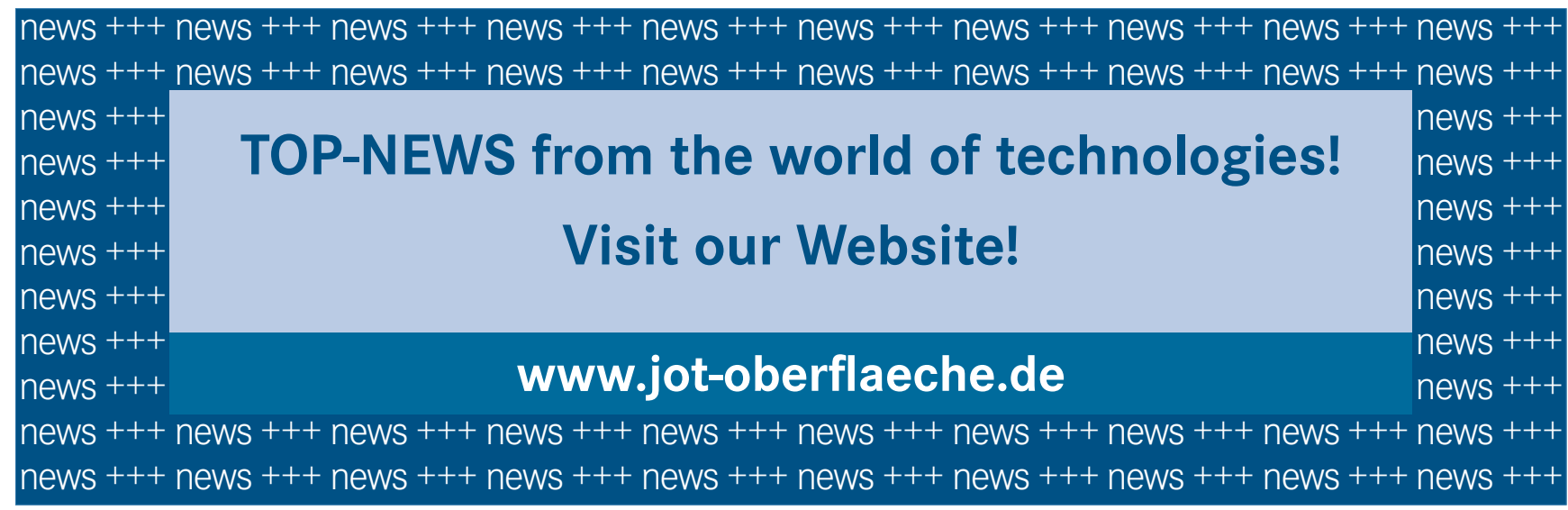

